\title{
Mapping and resolving galaxy formation at its peak epoch with Mahalo-Subaru and Gracias-ALMA
}

\author{
Tadayuki Kodama ${ }^{1,2,3}$, Masao Hayashi ${ }^{1}$, Yusei Koyama ${ }^{4}$, \\ Ken-ichi Tadaki ${ }^{1}$, Ichi Tanaka ${ }^{2}$, Rhythm Shimakawa ${ }^{2,3}$, \\ Tomoko Suzuki ${ }^{1,3}$ and Moegi Yamamoto ${ }^{1,3}$
}

${ }^{1}$ Optical and Infrared Astronomy Division, National Astronomical Observatory of Japan, Mitaka, Tokyo 181-8588, Japan email: t.kodama@nao.ac.jp

${ }^{2}$ Subaru Telescope, National Astronomical Observatory of Japan, 650 North A'ohoku Place, Hilo, HI 96720, USA

${ }^{3}$ Department of Astronomical Science, Graduate University for Advanced Studies, Mitaka, Tokyo 181-8588, Japan

${ }^{4}$ Institute of Space Astronomical Science, Japan Aerospace Exploration Agency Sagamihara, Kanagawa, 252-5210, Japan

\begin{abstract}
MAHALO-Subaru (MApping HAlpha and Lines of Oxygen with Subaru) project aims to investigate how the star forming activities in galaxies are propagated as a function of time, mass, and environment. It employs a unique set of narrow-band filters on MOIRCS/Subaru to search for Ha emitters associated to the proto-clusters or in narrow redshift slices in the general field. We have shown not only filamentary/clumpy structures of all the proto-clusters but also very high star formation activities therein especially at $z>2$. HST images from the CANDELS survey have revealed that nearly half of the $\mathrm{H} \alpha$ emitters in the field at $z \sim 2$ have clumpy structures. Among them, "red dusty clumps" are preferentially found at or near the mass center of galaxies. Therefore, they are probably linked to the formation of bulge component. To explore physical states and the mode of star formation of those forming galaxies, we have started Gracias-ALMA project in full coordination with the Mahalo-Subaru project. We will resolve molecular gas contents and dusty star formation within these galaxies, and tell whether clumps are formed by gravitational instability of gas rich disks, and whether bulges are formed by clump migration or through galaxy-galaxy mergers.
\end{abstract}

Keywords. galaxies: clusters, galaxies: formation, galaxies: evolution

\section{Mahalo-Subaru: Mapping star formation at its peak epoch}

It is widely recognised that the cosmic star formation rate (SFR) density and the AGN number density both show peaks at $z \sim 2$ (e.g. Hopkins \& Beacom 2006). Also galaxy formation should be dependent on environment. Our previous Subaru observations have revealed that the star formation activity in the cluster core at $z \sim 1.5$ is as high as that in the field, and that the peak of star forming activity is shifted outwards of clusters as time progresses (e.g. Hayashi et al. 2010; Koyama et al. 2011). We want to understand how the star forming activities in high density regions at high redshifts are intrinsically biased. how they are externally affected by surrounding environments, how the peak activity is shifted outwards to lower density environments with time, and what physical processes are involved and responsible for these phenomena.

To address those critical issues, we have been conducting the "Mahalo-Subaru" project (MApping HAlpha and Lines of Oxygen with Subaru; Kodama et al. 2013), a large 


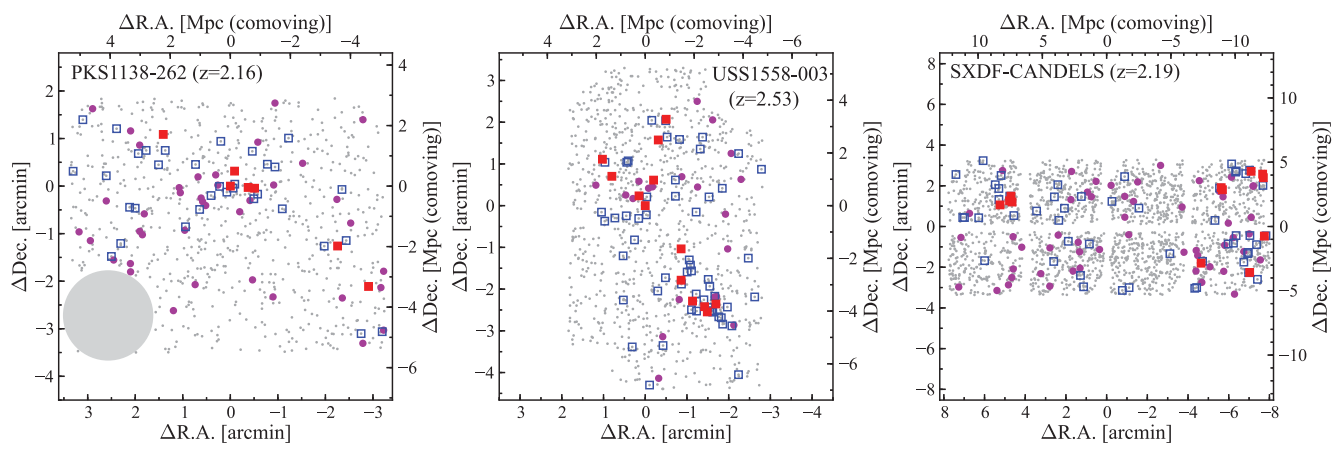

Figure 1. 2-D maps of two proto-clusters, USS1558-003 at $z=2.53$ (Hayashi et al. 2012), and PKS1138-262 at $z=2.16$ (Koyama et al. 2013), and a redshift slice $(z=2.09$ ) of a general field, SXDF-UDS-CANDELS (Tadaki et al. 2013). The red filled circles and blue open squares indicate HAE candidates associated to the proto-cluster separated at $\left.J-K_{\mathrm{s}}\right)=2.3$.

part of which was conducted as a Subaru open-use intensive program. Our targets are clusters/proto-clusters at $0.4<z<3.13$, and an un-biased general field SXDF-UDSCANDELS (4 redshift slices at $2.19<z<3.63$ ) (Table 1). We employ unique sets of narrow-band (NB) filters on the two wide-field cameras, Suprime-Cam (optical; $34^{\prime} \times 27^{\prime}$ ) and MOIRCS (NIR; $\left.7^{\prime} \times 4^{\prime}\right)$. Most of the MOIRCS NB filters are designed and manufactured specifically to our targets, and their wavelengths perfectly match to redshifted $\mathrm{H} \alpha$ or [OIII] lines from our $z>1.5$ targets. The Suprime-Cam NB filters in the $z^{\prime}$-band are also used to search for [OII] emitters at $z=1.46-1.62$. The line-of-sight velocity range that fall within the filter FWHM with respect to the cluster center is optimal $( \pm 1000$ $3000 \mathrm{~km} / \mathrm{s}$ ).

Using these NB filters, we have successfully identified lots of star-forming galaxies very efficiently in narrow redshift slices associated to the clusters or in the general field, which show nebular emission lines from ionized star-forming regions. We are sure about their membership to the clusters by the presence of emission lines and their SEDs based on colour-colour diagrams or photometric redshifts. We have completed most of the imaging observations under excellent conditions. Exposure times were typically 1-1.5hrs per broad-band, and 3-4hrs per narrow-band for $z \gtrsim 1.5$ targets. Figure 1 shows three best examples; two proto-clusters associated to the radio galaxies and the general field. We have discovered complex substructures traced by $\mathrm{H} \alpha$ emitters (HAEs), in the vicinity of, and physically associated the radio galaxies. It clearly indicates that these systems are still in a vigorous assembly phase. Star formation activity is also very intense even in the core regions of the proto-clusters. This is in contrast to lower redshift clusters where star formation activities are very low and the peak area of star formation activity is shifted outwards. It shows that cluster formation takes place in an inside-out fashion (Fig. 2).

It is notable that some of the HAEs show very red colours in $J-K_{s}(>2.3$ in Vegamag), indicating that they are dusty star forming galaxies (SFGs). Interestingly, those red HAEs tend to favor high density regions such as clumps and filaments along the structures. Therefore we expect that these are the key populations under the influence of environmental effects (Koyama et al. 2013).

These proto-clusters are very likely the sites where early-type galaxies, which will eventually dominate a rich cluster by the present-day, were just in their formation phase. Therefore, they serve us unique, excellent targets to investigate in detail the physical mechanisms of galaxy formation and its early evolution in dense environment. 
Table 1. A target list of the Mahalo-Subaru survey. S-Cam means Suprime-Cam.

\begin{tabular}{|c|c|c|c|c|c|c|c|}
\hline $\begin{array}{c}\text { environ- } \\
\text { ment }\end{array}$ & target & $z$ & line & $\begin{array}{c}\lambda \\
(\mu \mathrm{m})\end{array}$ & camera & NB & st \\
\hline $\begin{array}{l}\text { Lon } \\
\text { clus }\end{array}$ & $\begin{array}{l}52 \\
13 \\
09 \\
-6708\end{array}$ & $\begin{array}{r}395 \\
607 \\
41 \\
313\end{array}$ & $\begin{array}{l}\mathrm{H} \alpha \\
\mathrm{H} \alpha \\
\mathrm{H} \alpha \\
\mathrm{H} \alpha \\
{[\mathrm{O} \mathrm{II}]}\end{array}$ & $\begin{array}{l}0.916 \\
0.923 \\
1.011 \\
1.190 \\
0.676\end{array}$ & $\begin{array}{l}\text { S-Cam } \\
\text { S-Cam } \\
\text { S-Cam } \\
\text { MOIRCS } \\
\text { S-Cam }\end{array}$ & $\begin{array}{c}\text { NB912 } \\
\text { NB921 } \\
\text { NB1006 } \\
\text { NB1190 } \\
\text { NA671 }\end{array}$ & $\begin{array}{l}\text { Kodama+'04 } \\
\text { Koyama+'11 } \\
\text { not yet } \\
\begin{array}{l}\text { Koyama+'10 } \\
\text { observed }\end{array}\end{array}$ \\
\hline $\begin{array}{l}\text { High- } z \\
\text { cluster }\end{array}$ & \begin{tabular}{|l|} 
XCSJ2215-1738 \\
4C65.22 \\
CL0332-2742 \\
ClGJ0218.3-0510
\end{tabular} & $\begin{array}{l}1.457 \\
1.516 \\
1.61 \\
1.62\end{array}$ & $\begin{array}{c}{[\mathrm{OHI}]} \\
\mathrm{H} \alpha \\
{[\mathrm{OII}]} \\
{[\mathrm{OHI}]}\end{array}$ & $\begin{array}{l}0.916 \\
1.651 \\
0.973 \\
0.977\end{array}$ & $\begin{array}{l}\text { S-Cam } \\
\text { MOIRCS } \\
\text { S-Cam } \\
\text { S-Cam }\end{array}$ & $\begin{array}{c}\text { NB912/921 } \\
\text { NB1657 } \\
\text { NB973 } \\
\text { NB973 }\end{array}$ & $\begin{array}{l}\text { Hayashi+'10 } \\
\text { Koyama+'14 } \\
\text { observed } \\
\text { Tadaki+'12 }\end{array}$ \\
\hline $\begin{array}{l}\text { Proto- } \\
\text { cluster }\end{array}$ & $\begin{array}{l}4 \mathrm{C} 23.56 \\
\text { USS } 1558-003 \\
\text { MRC } 0316-257\end{array}$ & $\begin{array}{l}2.156 \\
2.483 \\
2.527 \\
3.130\end{array}$ & $\begin{array}{c}\mathrm{H} \alpha \\
\mathrm{H} \alpha \\
\mathrm{H} \alpha \\
{[\mathrm{OII}]} \\
{[\mathrm{O} I \mathrm{III}]}\end{array}$ & $\begin{array}{l}2.071 \\
2.286 \\
2.315 \\
2.539 \\
2.068\end{array}$ & $\begin{array}{l}\text { MOIRCS } \\
\text { MOIRCS } \\
\text { MOIRCS } \\
\text { MOIRCS }\end{array}$ & $\begin{array}{l}\text { NB2071 } \\
\text { CO } \\
\text { NB2315 } \\
\text { NB } 1550 \\
\text { NB2071 }\end{array}$ & $\begin{array}{l}\text { Koyama+'12 } \\
\text { Tanaka+'11 } \\
\text { Hayashi+'12 } \\
\text { not yet } \\
\text { not yet }\end{array}$ \\
\hline $\begin{array}{l}\text { General } \\
\text { field }\end{array}$ & $\begin{array}{l}\text { GOODS-N } \\
\left(70 \operatorname{arcmin}^{2}\right) \\
\text { SXDF-CANDELS } \\
\left(92 \operatorname{arcmin}^{2}\right)\end{array}$ & $\begin{array}{l}2.19 \\
2.53 \\
3.17 \\
3.63\end{array}$ & $\begin{array}{c}\mathrm{H} \alpha \\
{[\mathrm{OII}]} \\
\mathrm{H} \alpha \\
\mathrm{H} \alpha \\
{[\mathrm{O} \text { III] }} \\
{[\mathrm{OIII}]}\end{array}$ & $\begin{array}{l}2.094 \\
1.189 \\
2.094 \\
2.315 \\
2.093 \\
2.317\end{array}$ & $\begin{array}{l}\text { MOIRCS } \\
\text { MOIRCS } \\
\text { MOIRCS } \\
\text { MOIRCS } \\
\text { MOIRCS } \\
\text { MOIRCS }\end{array}$ & $\begin{array}{l}\text { NB2095 } \\
\text { NB1190 } \\
\text { NB2095 } \\
\text { NB2315 } \\
\text { NB2095 } \\
\text { NB2315 }\end{array}$ & $\mid \begin{array}{l}\text { Tadaki+'11 } \\
\text { observed } \\
\text { Tadaki+'13 } \\
\text { Tadaki+'13 } \\
\text { Suzuki+'14 } \\
\text { Suzuki+'14 }\end{array}$ \\
\hline
\end{tabular}

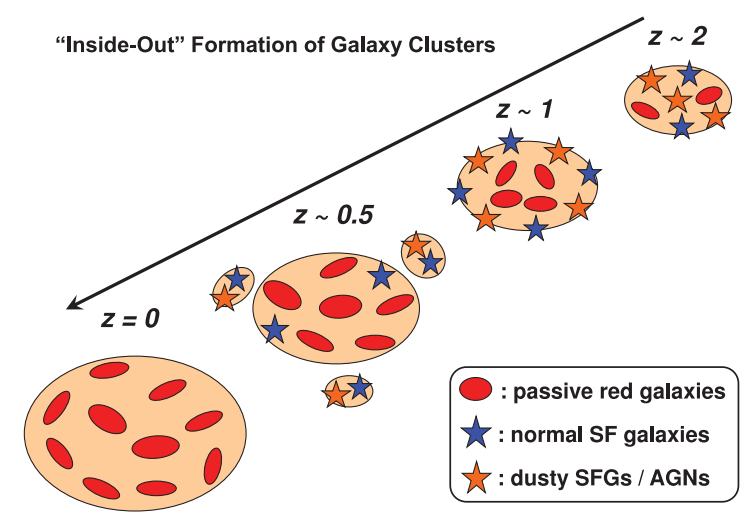

Figure 2. Schematic diagram of the inside-out evolution of clusters over cosmic times.

Deep optical-NIR HST images with high spatial resolution $\left(0.1-0.16^{\prime \prime}\right)$ are available in the SXDF-UDS-CANDELS field (Grogin et al. 2011). From those images we find that $40 \%$ of our HAEs at $z \sim 2$ show clumpy structures. Interestingly, massive clumpy galaxies tend to have a "red dusty clump" at or near the mass center of galaxies. Those clumpy galaxies may originate either from subgalaxy mergers or fragmentation of gas rich turbulent disks fed by cold streams of gas along the surrounding filamentary structures (Dekel et al. 2009). The red clumps may correspond to the merger of those clumps at galactic centers after they migrate to the centers due to dynamical friction (Bournaud et al. 2013). We may be witnessing the site of bulge formation in action. To test this hypothesis, we need to spatially resolve these clumpy galaxies and map out star formation activities within the galaxies. Such central dusty starburst, if present, must be identified.

We have also obtained deep spectra of HAE candidates in the two proto-clusters (PKS1138 and USS1558) with MOIRCS on Subaru to study physical properties of the SFGs in high density regions at high redshifts. The data have not only spectroscopically confirmed the membership of 63 galaxies (70\% of the observed targets) (Shimakawa 
et al. 2014a), but also provided us with their gaseous metallicities (Shimakawa et al. 2014b). We find that protocluster galaxies are more chemically enriched than those of field galaxies at a given stellar mass in the range of $\mathrm{M}_{\star} \lesssim 10^{11} \mathrm{M}_{\odot}$. There are two possible scenarios to acount for this interesting result. One is that due to higher gas pressure of intra-cluster medium, the gas once ejected from galaxies by outflows may fall back again and be recycled for further star formation, leading to more chemically enriched systems. The other is that metal poor gas that are loosely bounded to outer regions of galaxies may be selectively more stripped off due to tidal interaction or ram-pressure stripping, leading to higher averaged metallicity (Shimakawa et al. 2014b). Since the gas fraction for a given metallicity is expected to be different between the two scenarios, accurate measurement of molecular gas content by radio observations such as with ALMA will be the key to idetify the physical mechanism behind the environmental dependence of chemical evolution.

\section{Gracias-ALMA: Resolving gas and dust contents in SFGs}

Although we have identified many vigorously SFGs in our targets, we do not know the physical processes that are actually acting on the galaxies with the optical-NIR data alone. We therefore conduct the Gracias-ALMA project that is fully coordinated with the Mahalo-Subaru project. Gracias-ALMA stands for "GRAphing CO Intensity And Submm with ALMA". ALMA will provide us with (1) molecular gas content and (2) amount of hidden dusty star formation, and characterize the properties of SFGs. Furthermore, with ALMA, we can easily resolve individual galaxies on an angular scale of $0.1^{\prime \prime}$ which corresponds to $0.8 \mathrm{kpc}$ at $z=2.5$. Therefore we will be able to know where in galaxies dusty star formation and the dense molecular gas are distributed. For example, if the molecular gas content of the central red clump in the above clumpy galaxies is observed with ALMA, we can investigate the mode of star formation or star formation efficiency there which is expected to be different during a burst phase, providing a crucial test for the hypothesis of clumpy galaxy - bulge formation connection.

\section{References}

Bournaud, F., Perret, V., Renaud, F., et al. 2013, ApJ, 780, 57

Dekel, A., Birnboim, Y., Engel, G., et al. 2009, Nature, 457, 451

Grogin, N. A., Kocevski, D. D., Faber, S. M., et al. 2011, ApJS, 197, 35

Hayashi, M., Kodama, T., Koyama, Y., et al. 2010, MNRAS, 402, 1980

Hayashi, M., Kodama, T., Tadaki, K., et al. 2012, ApJ, 757, 15

Hopkins, A. M. \& Beacom, J. F. 2006, ApJ, 651, 142

Kodama, T., Balogh, M. L., Smail, I., et al. 2004, MNRAS, 354, 1103

Kodama, T., Tanaka, I., Hayashi, M., et al. 2013, ASPC, 476, 37

Koyama, Y., Kodama, T., Shimasaku, K., et al. 2010, MNRAS, 403, 1611

Koyama, Y., Kodama, T., Nakata, F., et al. 2011, ApJ, 734, 66

Koyama, Y., Kodama, T., Tadaki, K., et al. 2013, MNRAS, 428, 1551

Koyama, Y., Kodama, T., Tadaki, K., et al. 2014, ApJ, 789, 18

Shimakawa, R., Kodama, T., Tadaki, K., et al. 2014a, MNRAS, 441, L1

Shimakawa, R., Kodama, T., Tadaki, K., et al. 2014b, arXiv1406.5219

Suzuki, T., et al. 2014, in preparation

Tadaki, K., Kodama, T., Koyama, Y., et al. 2011, PASJ, 63, 437

Tadaki, K., Kodama, T., Ota, K., et al. 2012, MNRAS, 423, 2617

Tadaki, K., Kodama, T., Tanaka, I., et al. 2013, ApJ, 778, 114

Tanaka, I., De Breuck, C., Kurk, J., et al. 2011, PASJ, 63, 415 